\title{
Studies on Survival Analysis using Frailty Models under Bayesian Mechanism and its Further Scope
}

\author{
Jiten Hazarika ${ }^{1} \&$ Kuki Kalpita Mahanta ${ }^{2}$ \\ ${ }^{I}$ Professor, Department of Statistics, Dibrugarh University, Dibrugarh (Assam), India \\ ${ }^{2}$ Research Scholar, Department of Statistics, Dibrugarh University, Dibrugarh (Assam), India
}

\begin{abstract}
In this paper, an attempt has been made to discuss frailty models and their further developments under the Bayesian approach. The modeling of baseline hazard function with parametric and semiparametric approach, distribution of frailty variable along with derivation of posterior distribution with the help of the data likelihood and the prior distribution of the parameters of the frailty distribution with examples are also discussed here. Based on this review work it is observed that the Bayesian analysis of frailty models in survival analysis attains vast attention by the researchers. The study of frailty models under Bayesian mechanism has been continuing from various view points, but yet there is a vast scope of extending the Bayesian approach in survival modeling in presence of unobserved random effect (frailty) in different directions.
\end{abstract}

Keywords: Survival modeling, unobserved random effect, shared and correlated frailty models, Markov Chain Monte Carlo methods, Baseline Hazard function

\section{Introduction}

Frailty models, a specific area in survival analysis, provides a convenient way to introduce random effects, association and unobserved heterogeneity into models for survival data. In its simplest form, a frailty is an unobserved random proportionality factor that modifies the hazard function of an individual, or of related individuals. In essence, the frailty concept goes back to the work of Greenwood and Yule [1920] on "accident proneness". The term frailty itself was introduced by Vaupel et al. [1979] in univariate survival models and the model was substantially promoted by its application to multivariate survival data in a seminal paper by Clayton[1978] (without using the notation "frailty") on chronic disease incidence in families.

Normally in most clinical applications, survival analysis implicitly assumes a homogeneous population to be studied. This means that all individuals sampled into that study are subject in principle under the same risk (e.g., risk of death, risk of disease recurrence). In many applications, the study population cannot be assumed to be homogeneous but must be considered as a heterogeneous sample i.e., a mixture of individuals with different hazards. For example, in many cases it is impossible to measure all relevant covariates related to the disease of interest, sometimes because of economical reasons, sometimes the importance of some covariates is still unknown. The frailty approach is a statistical modeling concept which aims to account for heterogeneity, caused by unmeasured covariates. In statistical terms, a frailty model is a random effect model for time to event data, where the random effect (the frailty) has a multiplicative effect on the baseline hazard function. A frailty model is a multiplicative hazard model consisting of three components: a frailty (random effect), a baseline hazard function (parametric or non parametric), and a term modeling the influence of observed covariates (fixed effects).

Bayesian approach is a method of statistical inference in which Bayes' theorem is used to update the probability for a hypothesis as evidence is acquired. The Bayesian Mechanism refers to prior, posterior and predictive distributions to obtain estimates, compare models and test hypothesis and make predictions conditional on an observed sample. The use of Bayesian mechanism in survival analysis is wide spread. Several researchers have been developing Bayesian methodologies to analyse survival data with different frailty models and prior processes. In this paper, an attempt has been made to discuss frailty models in the context of Bayesian analysis based on past literatures and their further developments. The paper ends with a brief discussion on scope for further study.

\section{The Frailty Model}

Frailty models are extensions of population hazards model which is best known as the Cox model [Cox, 1972], a widely pursued model in survival analysis. According to Cox the hazard rate of an individual is given by,

$$
\mu(t, X)=\mu_{0}(t) \exp \left(\beta^{T} X\right)
$$

Where $\mu_{0}(t)$ denotes the baseline hazard function, assumed to be unique for all individuals in the study population. $X$ is the vector of observed covariates and $\beta$ the respective vector of regression parameters to be 
estimated. The mathematical convenience of this model is based on the separation of the effects of aging in the baseline hazard $\mu_{0}(t)$ from the effects of the covariates in the parametric term $\exp \left(\beta^{T} X\right)$.

Frailty models extends the Cox model such that the hazard of an individual depends in addition on an unobservable random variable $Z$, which acts multiplicatively on the baseline hazard function $\mu_{0}(t)$,

$$
\mu(t, Z, X)=Z \mu_{0}(t) \exp \left(\beta^{T} X\right) \ldots \ldots \ldots \ldots \ldots \ldots(2.2)
$$

Here frailty $Z$ is the random variable varying over the population decreases $(Z<1)$ or increases $(Z>1)$ the individual risk. The most important point here is that the frailty is unobservable. The respective survival function $S$, describing the fraction of surviving individuals in the study population is given by

$$
S(t \mid Z, X)=\exp \left(-Z \int_{0}^{1} \mu_{0}(s) d s \exp \left(\beta^{T} X\right)\right) .
$$

The cumulative hazard function is given by

$$
M(t)=\int_{0}^{t} \mu(s) d s .
$$

\section{Classification of Frailty Models}

The frailty models can be classified into two broad classes,

1) Univariate frailty Models: Models with an univariate survival time as endpoint and

2) Multivariate frailty Models: Models which describe multivariate survival endpoints (e.g., competing risk, recurrence of events in the same individuals, occurrence of a disease in relatives)

\subsection{Univariate Frailty Models}

In case of univariate frailty models a univariate (independent) lifetime is used to describe the influence of unobserved covariates in a proportional hazards model (heterogeneity). The variability of survival data is split into a part that depends on risk factor, and is therefore theoretically predictable, and a part that is initially unpredictable, even when all relevant information is known. A separation of these two sources of variability has the advantage that heterogeneity can explains some unexpected results or give an alternative interpretation of some results, for example, crossing-over effects or convergence of hazard functions of two different treatment arms [Manton and Stallard 1981] or leveling-off effects - that means the decline in the increase of mortality rates, which could result in a hazard function at old ages parallel to the x-axis [Aalen and Tretli 1999]

\subsection{Multivariate Frailty Models}

In case of multivariate frailty models multivariate survival data are used. Such kind of data occurs for example if lifetimes (or time of onset of a disease) of relatives (twins, parent child) or recurrent events like infections in the same individual are considered. Multivariate survival times are considered for the dependence in clustered event times, for example in the lifetime of patients in study centers in a multi-centre clinical trial, caused by centre-specific conditions [Andersen et al., 1999]. A natural way to model dependence of clustered event times is through the introduction of a cluster-specific random effect - the frailty. In other words, the lifetimes are conditional independent, given the frailty. This approach can be used for survival times of related individuals like family members or recurrent observations on the same person.

Let $S\left(t_{1} \mid Z, X_{1}\right)$ and $S\left(t_{2} \mid Z, X_{2}\right)$ be the conditional survival function of two related individuals with different vectors of observed covariates $X_{1}$ and $X_{2}$ respectively. Averaging over an assumed distribution for the latent variables (e.g., using a gamma, log-normal, stable distribution) then induce a multivariate model for the observed data. In the case of paired observations, the two dimensional survival function is of the form

$$
S\left(t_{1}, t_{2}\right)=\int_{0}^{\infty} S\left(t_{1} \mid z, X_{1}\right) S\left(t_{2} \mid z, X_{2}\right) g(z) d z .
$$

Where $g$ (.) denotes the density of the frailty $Z$. In the case of twins, $S\left(t_{1}, t_{2}\right)$ denotes the fraction of twins pair with twin 1 surviving $t_{l}$ and twin 2 surviving $t_{2}$.

Frailty models for multivariate survival data are derived under conditional independence assumption by specifying latent variables that act multiplicatively on the baseline hazard.

\section{Shared and Correlated Frailty Models}

There are two types multivariate frailty models available in survival analysis,-

$>$ Shared frailty model

$>$ Correlated frailty model

The shared frailty model is relevant to event times of related individuals, similar organs and repeated measurements. Individuals in a cluster are assumed to share the same frailty that's why this model is called shared frailty model. The survival times are assumed to be conditional independent with respect to the shared (common) frailty. For example consider the case of groups with pairs of individuals (bivariate failure times, e.g., event times of twins or parent-child).

Conditional on the frailty $Z$, the hazard function of an individual in a pair is of the form $Z$ $\mu_{0}(t) \exp \left(\beta^{T} X\right)$, where the value of $Z$ is common to both individuals in the pair, and thus is the cause for 
dependence between survival times within pairs. Independence of the survival times within a pair corresponds to a degenerate frailty distribution $\left(Z=1, \sigma^{2}=0\right)$. In all other cases with $\sigma^{2}>0$ dependence is positive by construction of the model. Conditional on $Z$, the bivariate survival function is given as

$$
S\left(t_{1}, t_{2} \mid Z\right)=S_{1}\left(t_{1}\right)^{z} S_{2}\left(t_{2}\right)^{z}
$$

In most applications it is assumed that the frailty distribution (i.e. the distribution of the random variable $Z$ ) is a gamma distribution with mean 1 and variance $\sigma^{2}$. Averaging the conditional survival function produces under this assumption survival functions of the form

$$
\mathrm{S}\left(\mathrm{t}_{1}, \mathrm{t}_{2}\right)=\left(\mathrm{S}_{1}\left(\mathrm{t}_{1}\right)^{-\sigma^{2}}+\mathrm{S}_{2}\left(\mathrm{t}_{2}\right)^{-\sigma^{2}}-1\right)^{1 / \sigma^{2}}
$$

Shared frailty explains correlations between subjects within clusters. However it does have some limitations (See Winke [2003]) also.

The correlated frailty models were developed for the analysis of bivariate failure time data, in which two associated random variables are used to characterize the frailty effect for each pair. For example, one random variable is assigned for partner 1 and one for partner 2 so that they would no longer be constrained to have a common frailty. These two variables are associated and have a joint distribution. Knowing one of them does not necessarily imply knowing the other. There is no more a restriction on the type of correlation. These two variables can also be negatively associated, which would induce a negative association between survival times. Assuming gamma frailties, Yashin and Iachine(1995) used the correlated gamma frailty model resulting in a bivariate survival distribution of the form

$$
S\left(t_{1}, t_{2}\right)=\frac{S_{1}\left(t_{1}\right)^{1-\rho} S_{2}\left(t_{2}\right)^{1-\rho}}{\left(S_{1}\left(t_{1}\right)^{-\sigma^{2}}+S_{2}\left(t_{2}\right)^{-\sigma^{2}}-1\right)^{\rho / \sigma^{2}}} \ldots
$$

One important problem in the area of frailty model is the choice of frailty distribution. The frailty distributions most often applied are the gamma distribution (Clayon [1978]; Vaupel et al.[ 1979]), the positive stable distribution (Hougaard [1986a]), a three-patameter distribution (PVF) (Hougaard [1986b], the compound poissson distribution (Aalen [1988,1992] and the log -normal distribution (McGilchrist and Aisbett, [1991]).

\section{Bayesian Approach in Frailty Models}

Bayesian approaches to frailty models with recent advances are computationally feasible in computing technology. Bayesian analysis of survival data using semiparametric models started immediately after Cox [1972] with work of Ferguson [1973] and Kalbfleisch [1978].

\subsection{Hazard Function Modeling}

Under Bayesian approach a parametric as well as a semiparametric approach is possible for modeling the baseline hazard function. In the parametric case, we can model the baseline hazard function parametrically. Sahu et al.[1997] used Weibull distribution to model the baseline hazard function and they compared it with the flexible baseline hazard model based on correlated prior process.

Let us consider the survival time of $i^{\text {th }}$ individual $(i=1,2, \ldots n)$ be $T_{i}$ and given the unobserved frailty parameter denoted by $\mathrm{z}_{\mathrm{i}}$ (for the $i^{\text {th }}$ individual), the hazard function as given in equation (2.2) is as follows:

$$
\mu\left(t_{i} \mid x_{i}, z_{i}\right)=\mu_{0}\left(t_{i}\right) \exp \left(\beta^{T} x_{i}\right) z_{i}
$$

Where $x_{i}$ is the fixed covariate vector, $\beta$ is the regression parameter and $\mu_{0}($.$) is the baseline hazard$ function and $z_{i}$ is the frailty parameter for the $i^{t h}$ individual.

In the parametric approach we can model the baseline hazard function parametrically. For example if we use weibull distribution to model it the weibull baseline hazard function is given by

$$
\mu_{0}\left(t_{i}\right)=\lambda \gamma t_{i}^{\gamma-1}, i=1,2, \ldots n \ldots \ldots \ldots \ldots \ldots(5.1 .2)
$$

Where $\lambda, \gamma>0 ; \lambda$ and $\gamma$ are unknown hypermeters.

Now from equation (5.1.1) the hazard of the $i^{t h}$ individual is given by

$$
\mu\left(t_{i}\right)=\lambda \gamma t_{i}^{\gamma-1} \exp \left(\beta^{T} x_{i}\right) z_{i}
$$

The cumulative hazard function is given by

$$
M_{i}(t)=\lambda t_{i}^{\gamma} \exp \left(\beta^{T} x_{i}\right) z_{i}
$$

Then the survival function is given by

$$
S_{i}(t)=\exp \left(-M_{i}(t)\right)=\exp \left(-\lambda t_{i}^{\gamma} \exp \left(\beta^{T} x_{i}\right) z_{i}\right)
$$

Let us consider right censored survival data $\left(t_{i}, \delta_{i}\right), i=1,2, \ldots, n$ and assume that the censoring is non informative. Let $\delta_{i}$ be the indicator variable taking value 1 if the $i^{\text {th }}$ individual fails and value 0 otherwise. Hence $t_{i}$ is a failure if $\delta_{i}=1$ and censoring time otherwise. Hence the triplet $\left(t_{i}, \delta_{i}, x_{i}\right), i=1,2, \ldots, n$ is observed for all $\mathrm{n}$ individuals. Given the unobserved frailty $z_{i}, t_{i}$ 's are independent. Hence the complete data likelihood is given by 


$$
\begin{aligned}
\mathrm{L}(\text { parameter, data })= & \prod_{i=1}^{n}\left[\mu\left(t_{i}\right)\right]^{\delta_{i}} S_{i}(t) \\
& =\prod_{i=1}^{n}\left[\lambda \gamma \mathrm{t}_{\mathrm{i}}{ }^{\gamma-1} \exp \left(\beta^{\mathrm{T}} x_{i}\right) z_{i}\right]^{\delta_{i}} \exp \left(-\lambda \mathrm{t}_{\mathrm{i}}{ }^{\gamma} \exp \left(\beta^{\mathrm{T}} x_{i}\right) z_{i}\right) .
\end{aligned}
$$

Again recent advances in Bayesian nonparametric survival analysis give us wide range of choices for the prior processes to model the baseline hazard function. Modeling the baseline hazard using prior processes is very common; see Sinha and Day [1997] for more details.

As for example let us consider a piecewise exponential model. Piecewise exponential models and prior processes on the components provide a very flexible framework for modeling univariate survival data. Let $\left\{\left(t_{i}, \delta_{i}\right.\right.$ ,$\left.\left.x_{i}\right), i=1,2, \ldots, n\right\}$ be the observed data. We divide time into g prespecified interval $I_{k}=\left(t_{k-1}, t_{k}\right)$ for $k=1,2, \ldots, g$ where $0=t_{0}<t_{1}<\ldots<t_{g}<\infty, t_{g}$ being the last survival or censored time and assume that the baseline hazard to be constant within each interval. i.e,

$$
\mu_{0}\left(t_{i}\right)=\mu_{k, \text { for }} t_{i} \epsilon I_{K}
$$

To correlate the $\mu_{\mathrm{k}}$ 's in adjacent intervals, a discrete-time martingale process is used by Arjas and Gasbarra[1994] for univatiate survival models. Given $\left(\mu_{1}, \mu_{2}, \ldots, \mu_{k}\right)$ they specify that

$$
\mu_{k \mid} \mu_{1}, \mu_{2}, \ldots, \mu_{k-1} \sim \operatorname{Gamma}\left(\alpha_{k}, \frac{\alpha_{k}}{\lambda_{k-1}}\right), k=1,2, \ldots, g .
$$

Where $\mu_{0}=1$. The parameter $\alpha_{k}$ controls the amount of smoothness available.

The corresponding hazard and cumulative hazard function for the $i^{\text {th }}$ individual is given by

$$
\mu\left(t_{i}\right)=\mu_{k} \exp \left(\beta^{T} x_{i}\right) z_{i}
$$

$$
M_{i}(t)=\mu_{k} \exp \left(\beta^{T} x_{i}\right) z_{i} \Delta_{k}
$$

$$
\text { Where } \Delta_{k}=\left\{\begin{array}{c}
0, \text { if } t_{i}<t_{k-1} \\
t_{i}-t_{k-1}, \text { if } t_{k-1} \leq t_{i}<t_{k} \\
t_{k}-t_{k-1}, \text { if } t_{i} \geq t_{k}
\end{array}\right.
$$

If the $i^{\text {th }}$ individual survived beyond the $k^{\text {th }}$ interval i.e, $t_{i} \geq t_{k}$ then the likelihood contribution is given by

$\left(\mu_{\mathrm{k}} \exp \left(\beta^{\mathrm{T}} x_{i}\right) z_{i}\right)^{0} \exp \left(-\mu_{\mathrm{k}} \exp \left(\beta^{\mathrm{T}} x_{i}\right) z_{i}\left(t_{k}-t_{k-1}\right)\right)=\exp \left(-\mu_{\mathrm{k}} \exp \left(\beta^{\mathrm{T}} x_{i}\right) z_{i}\left(t_{k}-t_{k-1}\right)\right)$

When $t_{k-1}<t_{i}<t_{k}$ the likelihood contribution is given by

$$
\left(\mu_{\mathrm{k}} \exp \left(\beta^{\mathrm{T}} x_{i}\right) z_{i}\right)^{\delta_{i}} \exp \left(-\mu_{\mathrm{k}} \exp \left(\beta^{\mathrm{T}} x_{i}\right) z_{i}\left(t_{i}-t_{k-1}\right)\right)
$$

Hence we arrive at the following complete data likelihood

$$
\prod_{i=1}^{n}\left\{\prod_{k=1}^{g_{i}} \exp \left(-\mu_{\mathrm{k}} \exp \left(\beta^{\mathrm{T}} x_{i}\right) z_{i}\left(t_{k}-t_{k-1}\right)\right)\right\}\left(\mu_{g_{i}+1} \exp \left(\beta^{\mathrm{T}} x_{i}\right) z_{i}\right)^{\delta_{i}} \exp \left(-\mu_{g_{i}+1} \exp \left(\beta^{\mathrm{T}} x_{i}\right) z_{i}\left(t_{i}-t_{g_{i}}\right)\right)
$$

Where $g_{i}$ is such that $t_{i} \in\left(t_{g_{i}}, t_{g_{i}+1}\right)=I_{g_{i}+1}$

Kalbflesich [1978] and Ferguson and Phadia [1979] introduced the gamma prior process for non parametric Bayesian analysis of right censored continuous survival data. Again Sari et al[2014] used gamma prior process to the recurrence of infection time data at the point of catheter insertion for kidney patients using portable equipment. Nieto-Barajas and Yin [2008] took a mixture prior of a Markov gamma process for full Bayesian analysis of a bone marrow transplant data set. Burridge [1981], Clayton [1991] used Levy process with positive independent increments in disjoint intervals to model the prior process of cumulative hazard. Gamerman [1991] presented a linear Bayesian method to analyze univariate survival data with possible right censoring using the auto-correlated prior process. Sinha[1998] also used an auto-correlated incomplete prior process for the baseline hazard and a joint prior for unknown regression parameter and variance (independent of the prior process) in the conditional proportional hazard model. Sinha[1993] presented the Bayesian semiparametric conditional poisson process model for multiple event-time data. Ferguson and Phadia[1979] and Iorio et al. [2009] worked on Bayesian non parametric survival analysis using Dirichlet prior process. Pennell and Dunson[2006] also used the Dirichlet prior process for a semiparametric shared frailty and for multiplicative innovation on the frailty for multiple event time data. 


\subsection{The Frailty Distribution}

The frailty random variable $z_{i}$ 's are assumed to be independent and identically distributed for each individual having some parametric distribution with unit mean (to obtain identifiability). A few examples of frailty distributions are given in Clayton and Cuzick[1985], Oakes[1989]. The gamma distribution is most commonly used to model the frailty [Oakes, 1989; Clayton, 1991]. As for example Aslanidou et al.[1998] assumed that $z_{i}$ 's to be independent with

$$
z_{i} \sim \operatorname{Gamma}\left(k^{-1}, k^{-1}\right), i=1,2, \ldots, n \ldots \ldots \ldots \ldots(5.2 .1)
$$

so that $k$ is the unknown variance of $z_{i}$ 's and the density of Gamma $(a, b)$ is proportional to $x^{a-l} \exp (-b x)$. Thus, higher value of $k$ signifies greater heterogeneity among different individuals.

\subsection{Derivation of Posterior Distributions}

The Bayes theorem leads to the following joint posterior density function of the parameter vector $\omega$

$$
f(\omega \mid \text { data })=\frac{f(\text { data } \mid \omega) f(\omega)}{f(\text { data })}
$$

The Bayes theorem can also be applied to one of the parameters $\omega_{j}$ while conditioning on the other parameters. We will denote the parameter vector without the $j^{\text {th }}$ component $\omega_{j}$ by $\omega_{(-j)}$. For $\mathrm{j}^{\text {th }}$ component of $\omega$ the following conditional posterior density

$$
f\left(\omega_{j} \mid \text { data, } \omega_{(-j)}\right)=\frac{f(\text { data } \mid \omega) f\left(\omega_{j} \mid \omega_{(-j)}\right)}{f\left(\text { data } \mid \omega_{(-j)}\right)} .
$$

Furthermore, since the conditioning of the prior density of $\omega_{j}$ on the other parameters in not instrumental (we assume independence of prior densities) we drop here this type of conditioning. In the righthand side of the conditional posterior density (5.3.2) the denominator does not depend on $\omega_{j}$. therefore, the numerator on the right-hand side of (5.3.2) and the conditional posterior density of $\omega_{j}$ are proportional:

$$
f\left(\omega_{j} \mid \text { data, } \omega_{(-j)}\right) \propto f(\text { data } \mid \omega) f\left(\omega_{j}\right)
$$

using the likelihood functions (5.1.6) and (5.1.11) we will make use of the unnormalised conditional posterior densities of form (5.3.3) for all the parameters since the normalizing factor (i.e, the denominator on the righthand side of (5.3.2) is in most cases, difficult to obtain.

The data likelihood of the parameters in the frailty model can be obtained by integrating out the frailty variable $z_{i}$ 's from the likelihoods (5.1.6) and (5.1.11). The final forms of the data likelihoods after integration are too complicated to work with. Thus, it is not easy to evaluate the marginal posterior distributions of the parameters analytically. To circumvent this problem, we use a Markov chain Monte Carlo method Gibbs sampler, see e.g., Gelfand and Smith [1990] and Gilks et al. [1996] with the data augmentation method (Tanner and Wong, 1987) to generate samples from the appropriate marginal posterior distributions.

\subsection{MCMC Methods in Frailty Models}

Aslanidou et al.[1998] analysed a multivariate survival data from a Bayesian perspective using Markov Chain Monte Carlo(MCMC) methods. Mallick et al. [1999] used a Bayesian approach to model univariate and multivariate survival data with censoring. They described a MCMC algorithm to obtain the estimate of the hazard function as well as the survival curve. Walker and Mallick [1999] used a Bayesian semiparametric approach for accelerated failure time model and they described a MCMC algorithm to obtain a predictive distribution for a future observation given both uncensored and censored data. They explained how the resultant model is implemented via MCMC methods. Sinha and Maiti [2004] developed a Bayesian analysis based on a MCMC algorithm for the analysis of Panel- Count Data with Dependent Termination of a data set from a clinical trial. They used Bayesian approach using MCMC methods with the help of a comprehensive simulation study. Yin and Ibrahim [2006] used the MCMC algorithm for a real data set from a melanoma clinical trial. Again Basu and Tiwari [2010] developed a model that unifies the mixture cure and competing risk approaches and that can handle the masked causes of death in a natural way. In this development the Markov Chain sampling is used.

\section{Further Scope of the Study}

From the above discussion, it is observed that the Bayesian analysis of frailty models in survival analysis attains vast attention by the researchers. The study of frailty models under Bayesian mechanism has been continuing from various view points, but yet there is a vast scope of extending the Bayesian approach in survival modeling in presence of unobserved random effect (frailty) in different directions. Moreover, it is to be noted that the behavior of the unobserved covariates are different according to different socio-demographic variables, method of diagnosis of the disease and effect of correlated variables. 
So far our knowledge goes still there are some frailty models in survival analysis which are remained undiagnosed under Bayesian mechanism. For example some data arises in real life which is known to be symmetric often exhibits departure from symmetry i.e, presence of skewness. In most of the cases it is observed that skewness is also present in survival data. An ordinary method for reducing the skewness is the use of square root or logarithm transformations of responses. In most of the survival data sets, because of high rate of missingness, skewness is remained even after using the transformations. An alternative is to develop skew models (by introducing skewness) starting from symmetric models, which can effectively deal with introduced skewness in otherwise symmetric data. Skew normal distribution of Azzalini (1985) and its further extension by Chakraborty et al. [2015] are such kind of distributions (for more details about skewed distributions see Chakraborty and Hazarika [2011]). A few number of works are done using skewed distribution to model survival data. For example Callegaro [2012] used Log-skew-normal distribution to fit accelerated failure time models. Again Baghfalaki and Ganjali [2015] used a skew-normal mixed effect model for longitudinal measurements and a Cox proportional hazard model for time to event variable using a real HIV data set. Bayesian approach using Markov chain Monte Carlo is adopted by them for parameter estimation. Also Sahu and Day [2004] developed a class of log-skew-t distribution for the frailty. This class includes the log-normal distribution along many other heavy tailed distributions e.g. Cauchy or log-t as special cases. But, most of the above skewed distributions are not considered under Bayesian mechanism. So there is a vast scope to extend the work of frailty models to some skewed distributions under Bayesian mechanism.

\section{References}

[1] Aalen, O.O. Heterogeneity in survival analysis, Statistics in Medicin 7.1988. 1121-1137

[2] Aalen, O.O. Modelling Heterogeneity in Survival Analysis by the Compound Poisson Distribution, Annals of Applied Probability 4(2), 1992. 951-972.

[3] Aalen, O.O., Tretli, S. Analysing incidence testis cancer by means of a frailty model,Cancer Causes and Controll, 1999. 285-292.

[4] Andersen, P.K., Klein, J.P., Zhang, M. J. Testing for centre effects in multi-centre survival studies: A Monte Carlo Comparison of Fixed and Random Effect, Tests Statistics in Medicine 18, 1999.1489-1500

[5] Arjas, E. and Gasbarra, D., Nonparametric Bayesian inference from right censored survival data, using the Gibbs sampler. Statistica Sinica, 1994.505-524.

[6] Aslanidou, H., Dey D. K. and Sinha D. Bayesian analysis of multivariate survival data using Monte Carlo methods, The Canadian Journal of Statistics, 26(11),1998,33-48

[7] Azzalini, A. A class of distributions which includes the normal ones, Scandinavian journal of statistics, 1985 171-178.

[8] Basu, S. and Tiwari, R.C. Breast Cancer survival, competing risks and mixture cure model: a Bayesian analysis J.R. Statist. Soc. A 173, 2010, 307-329

[9] Baghfalaki, T., \& Ganjali, M. A bayesian approach for joint modeling of skew-normal longitudinal measure-ments and time to event dataRevstat Statistical Journal, 13(2), 2015, 169-191.

[10] Burridge, J. Empirical Bayes analysis of survival time data. Journal of the Royal Statistical Society B 43, 1981, 65-75.

[11] Callegaro, A. Log-skew-normal accelerated failure time models Working Paper Series, N. 3, March, 2012, Department of Statistical Sciences, University of Padua, Italy.

[12] Chakraborty, S., \& Hazarika, P. J. A survey of the theoretical developments in univariate skew normal distributions, Assam Statist. Rev,25(1),2011, 41-63.

[13] Chakraborty, S., Hazarika, P. J., \& Ali, M. M. A multimodal skewed extension of normal distribution: its properties and applications. Statistics, 49(4), 2015.859-877.

[14] Clayton, D.G. A model for association in bivariate life tables and its application in epidemiological studies of familial tendency in chronic disease incidence. Biometrika 65, 1978.141-151.

[15] Clayton, D. and Cuzick. J. Multivariate generalizations of the proportional hazards models. Journal of the Royal Statistical Society A 148, 1985,82-117.

[16] Clayton, D. A Monte Carlo Method for Bayesian inference in frailty models. Biometrics 47, 1991.467-48.

[17] Cox, D.R. Regression Models and Life-Tables. Journal of the Royal Statistical Society B 34, 1972.187-220.

[18] Ferguson, T.S.,A Bayesian analysis of some nonparametric problems. The annals of statistics, 1973.209-230.

[19] Ferguson, T.S. and Phadia, E.G. Bayesian nonparametric estimation based on censored data. Annals of Statistics 7, 1979.163-186.

[20] Gamerman, D.Dynamic Bayesian models for survival data. Applied Statistics 40, 1991, 63-79

[21] Gelfand, A. E., \& Smith, A. F. Sampling-based approaches to calculating marginal densities.Journal of the American statistical association, 85(410), 1990. 398-409.

[22] Gilks, W. R., Richardson, S., \& Spiegelhalter, D. J. Introducing Markov chain Monte Carlo. Markov chain Monte Carlo in practice 1, 1996,1-19.

[23] Greenwood, M., Yule, G. U. An inquiry into the nature of frequency distributions representative of multiple happenings with particular reference to the occurrence of multiple attacks of disease or repeated accidents. Journal of the Royal Statistical Society 83, 1920. 255-79.

[24] Hougaard, P. A class of multivariate failure time distribution. Biometrika,73,1986a,671-678

[25] Hougaard, P. Survival models for heterogeneous populations derived from stable distributions. Biometrika 73, 1986b, 671-678.

[26] Iorio, M.D., Johnson, W. O., Muller, P. and Rosner, G. L. Bayesian nonparametric nonproportional Hazards Survival Modelling. Biometrics 65, 2009. 762-771

[27] Kelbfleisch, J. D. Nonparametric Bayesian analysis o survival data. Journal of the Royal Statistical Society B 40, 1978.214-221.

[28] Mallick, B. K., Denison D. G. T. and Smith, A. F. M. Bayesian survival analysis using a MARS Model. Biometrics 55, 1999, 10711077

[29] [29] Manton, K.G. and Stallard, E. Methods for evaluating the heterogeneity of aging processes in human populations using vital Statistics data: explaining the black/ white mortality crossover by a model of mortality selection. Human Biology 53, 1981. 47-67.

[30] McGilchrist, C.A. and Aisbett, C.W. Regression with Frailty in Survival Analysis.Biometrics 47, 1991. $461-466$. 
[31] Nieto- Barajas L. E. and Yin G. Bayesian semiparametric cure rate model with an unknown threshold. Scandinavian Journal of Statistics, vol.35, 2008. 540-556

[32] Oakes, D. Bivariate survival models induced by frailties. Journal of American Statistical Association 84, 1989.487-493.

[33] Pennell, M. and Dunson D. B. Bayesian semiparametric Dynamic Frailty Models for Multiple Event Time Data. Biometrics 62, 2006.1044-1052

[34] Sahu, S.K., Dey, D.K., Aslannidou, H., and Sinha, D. A Weibull regression model with gamma frailties for multivariate survival data.. Lifetime Data Analysis 3, 1997. 123-137

[35] Sahu, S. K. and Dye, D. K. On multivariate survival models with a skewed frailty and a correlated baseline hazard process. Skewelliptical distributions and their applications: a journey beyond normality, 2004. 321-338.

[36] Sari, I.M., Thamrin, A.S. and Lawi, A. Piecewise exponential frailty models on survival data using Bayesian Approach.Conference paper: international conference on science (ICOS-1), 2014 Nov.

[37] Sinha, D. Semiparametric Bayesian analysis of multiple time data. Journal of American Statistical Association 88, 1993.979-983.

[38] Sinha, D. and Dey, D.K. Semiparametric Bayesian analysis of Survival data. Journal of American Statistical Association 92 , 1997. 1195-1212.

[39] Sinha, D. Posterior likelihood methods for multivariate survival data. Biometrics 54, 1998. 1463-1474.

[40] Sinha, D. and Maiti, T. A Bayesian approach for the analysis of panel-count data with dependent termination. Biometrics $60,2004$. 34-40.

[41] Tanner, M. A. and Wong, W. H. The calculation of posterior distributions by data augmentationJournal of the American statistical Association, 82(398), 1987. 528-540.

[42] Vaupel, J.W., Manton, K.G. and Stallard, E. The impact of Heterogeneity in Individual Frailty on the Dynamics of Mortality.Demography 16, 1979. 439-454.

[43] Walker, S. and Mallick, B.K. A Bayesian Semiparametric Accelerated Failure Time Model. Biometrics,55(2), 1999, 477-483

[44] Winke, A. Frailty models. Working paper of the Max Planck Institute for Demographic Research 2003.

[45] Yashin, A.I. and Iachine, I.A., Genetic analysis of durations: correlated frailty model applied to survival of Danish twins. Genetic epidemiology, 12(5), 1995.529-538.

[46] Yin G. and I brahim J. G. Bayesian transformation hazard models, $2^{\text {nd }}$ Lehmann Symposium- Optimlity, 49,2006.170-182 